\title{
Soziologische Revue
}

Besprechungen neuer Literatur

\author{
Begründet von Heinz Hartmann \\ Herausgegeben von \\ Richard Münch, Birgitta Nedelmann, \\ Gert Schmidt, Hans-Georg Soeffner
}

\begin{tabular}{lll}
\hline Jahrgang 24 & Heft 2 & April 2001
\end{tabular}

\section{Editorial}

Richard Münch

\section{Essay}

Walter M. Sprondel, Eine neue Flaschenpost. Untersuchungen zur

Frankfurter Schule

Sammelbesprechungen

Gerhard Himmelmann, Globalisierung und Demokratie

Wolfram Lamping / Friedbert W. Rüb, Sozialstaat und Globalisierung ......... 157

John P. Neelsen, Menschenrechte, Demokratie und Internationale Politik . 178 Roland Roth, Und sie bewegen sich doch. Neue Veröffentlichungen zur

Bewegungsforschung

\section{Einzelbesprechungen}

\section{GESELLSCHAFT}

Klaus J. Milich, Die frühe Postmoderne. Geschichte eines europäischamerikanischen Kulturkonflikts (Georg Kamphausen)

Wolfgang Lipp, Heimat - Nation - Europa. Wohin trägt uns der Stier?

Standorte in Bewegung (Justin Stagl)

Andreas Ziemann, Die Brücke zur Gesellschaft. Erkenntniskritische und topographische Implikationen der Soziologie Georg Simmels 


\section{MINDERHEITEN / MIGRATION}

Rainer Strobl / Wolfgang Kühnel, Dazugehörig und ausgegrenzt. Analysen zu Integrationschancen junger Aussiedler (Hartmut M. Griese)

Jörg Stolz, Soziologie der Fremdenfeindlichkeit. Theoretische und empirische Analysen (Lutz Hoffmann)

Elisabeth Beck-Gernsheim, Juden, Deutsche und andere Erinnerungs-

landschaften. Im Dschungel der ethnischen Kategorien (Hilde Weiss) . 214

Petrus Han, Soziologie der Migration. Erklärungsmodelle, Fakten,

Politische Konsequenzen, Perspektiven (Thomas Müller-Schneider) ..... 216

\section{SOZIALE PROBLEME}

Bertold Vogel, Ohne Arbeit in den Kapitalismus. Der Verlust der Erwerbs-

arbeit im Umbruch der ostdeutschen Gesellschaft (Frank Adler)

Gerhard Bäcker u.a., Sozialpolitik und soziale Lage in Deutschland

(Lutz Leisering)

Mirjam von Felten, ,... aber das ist noch lange nicht Gewalt".

Empirische Studien zur Wahrnehmung von Gewalt bei Jugendlichen

(Matthias Ulbrich-Herrmann)

VARIA

Ralf Ottermann, Soziologie des Betrugs (Günter Gutsche)

Andreas Dörner, Politische Kultur und Medienunterhaltung.

Zur Inszenierung politischer Identitäten in der amerikanischen

Film- und Fernsehwelt (Elmar J. Koenen)

Frithjof Zerger, Klassen, Milieus und Individualisierung. Eine empirische Analyse zum Umbruch der Sozialstruktur (Rolf Küttel /

Volker Bornschier)

\section{Bereichsrezensionen}

Einführungen (Thorsten Bonacker) ........................................................... 239

Umweltsoziologie (Jens Jetzkowitz) .......................................................... 243

Soziologie und Internet (Dirk vom Lehn) ………….................................... 247

Jugend (Steffen Malo / Uwe Sander) .......................................................... 250

Familie (Birgit Pfau-Effinger) …………………………………………….... 253

Wissenssoziologie (Michael Schwab-Trapp) …………………………………..... 257

AutorInnen des 2. Heftes ............................................................................ 261

Eingegangene Bücher .................................................................................. 262 


\section{GESCHÄFTLICHE MITTEILUNGEN}

\section{Geschäftsführender Herausgeber (verantwortlich): Prof. Dr. Richard Münch}

Redaktion: Carsten Stark, Annerose Baum, Tina Seidl

Anschrift der Redaktion: Universität Bamberg, Weidendamm 60, 96045 Bamberg

Rezensionsexemplare (jeweils zwei Exemplare) und Zuschriften sind an die Redaktion zu richten.

Unverlangt zugesandte Rezensionen werden aus grundsätzlichen Erwägungen nicht abgedruckt.

Bezugsbedingungen: Die Zeitschrift erscheint einmal im Vierteljahr (Januar, April, Juli, Oktober). Jahresabonnement: Inland DM 220,- (204,- + 16,- Versandspesen); Ausland DM 226,- (204,-- + 22,-- Versandspesen). Studentenabonnement sowie Abonnement für Mitglieder soziologischer Fachverbände (nur Inland) DM 114,- (DM 98,- + 16,- Versandspesen). Einzelheft DM 70,- zuzüglich Versandspesen. Die Preise enthalten bei Lieferung in EU-Staaten die Mehrwertsteuer, für das übrige Ausland sind sie Bruttopreise. Ermittlung der gebundenen Ladenpreise für Österreich und die Schweiz: Österreich: DM-Preis $\times 7,3=$ öS-Preis (ab 0,5 aufgerundet, bis 0,4 abgerundet auf volle Schillinge). Schweiz: DM-Preis $\times 0,88=s$ Fr-Preis (aufgerundet auf volle Franken).

Das Abonnement verlängert sich jeweils um ein Jahr, wenn es nicht spätestens zwei Monate vor Ablauf des Kalenderjahres gekündigt wird. Die Lieferung geschieht auf Kosten und Gefahr des Empfängers. Kostenlose Nachlieferung in Verlust geratener Sendungen erfolgen nicht.

Werbeanzeigen und Werbebeilagen besorgt der Verlag. Für den Anzeigenteil verantwortlich: Ulrike Staudinger.

Hinweis gemäß $§ 26$ Absatz 1, Bundesdatenschutzgesetz: Die Bezieher der SOZIOLOGISCHEN REVUE sind in einer Adreßdatei gespeichert, die mit Hilfe der automatisierten Datenverarbeitung geführt wird.

Die SOZIOLOGISCHE REVUE wird regelmäßig in den folgenden Informationsdiensten erfaßt: International Review of Publications in Sociology (Sociological Abstracts, P.O. Box 22206, San Diego, CA 92122, USA); Sozialwissenschaftliches Literaturinformationssystem SOLIS (Informationszentrum Sozialwissenschaften, Lennéstr. 30, 53113 Bonn).

Die in dieser Zeitschrift veröffentlichten Beiträge sind urheberrechtlich geschützt. Fotokopien für den persönlichen und sonstigen eigenen Gebrauch dürfen nur von einzelnen Beiträgen oder Teilen daraus als Einzelkopien hergestellt werden. Jede darüber hinausgehende Vervielfältigung bedarf der Genehmigung des Verlages und verpflichtet zur Gebührenzahlung.

Soziologische Revue im Internet

http://www.soziologische-revue.de

\section{OLDENBOURG WISSENSCHAFTSVERLAG GMBH}

„Gemäß unserer Verpflichtung nach $\$ 8$ Abs. 3 PresseG i.V.m. Art. 2 Abs. 1c DVO zum BayPresseG geben wir die Inhaber und Beteiligungsverhältnisse am Verlag wie folgt an:

Oldenbourg Wissenschaftsverlag GmbH, Rosenheimer Str. 145, 81671 München. Alleiniger Gesellschafter des Verlages ist die R. Oldenbourg Verlag GmbH unter der gleichen Anschrift. Alleiniger Gesellschafter der R. Oldenbourg Verlag GmbH ist die R. Oldenbourg GmbH \& Co. KG, ebenfalls unter der gleichen Anschrift."

Druck: R. Oldenbourg, Graphische Betriebe Druckerei GmbH, Hürderstraße 4, 85551 Kirchheim

Satz: Falkner GmbH, 82266 Inning/A. 


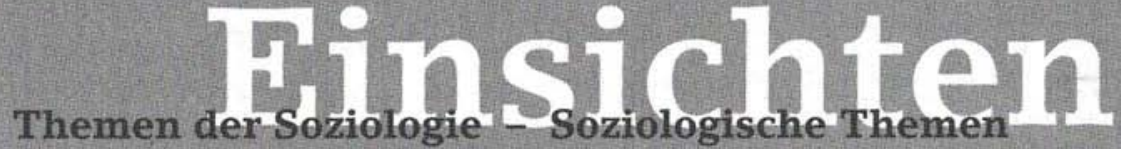 Unsere Neuen}

\section{GUNNAR STOLLBERG}

\section{Medizinsoziologie}

Die Medizinsoziologie ist eine Bindestrich-Soziologie im

Ubergang. Erst in den $1950 e$ / 1960 er Jahren etabliert, gehört sie in Deutschland seit $197^{\circ}$ zur Pflichtlekture in der vorkdinischen medizinischen Ausbildung. Gegenwartig differenzieren sich Soziologien der Gesundheit, des Körpers sowie des Sterbens und des Todes aus. In dieser Situation setzt der vorliegende Band die Medizinsoziologie sowohl ats spezielle Soziologie in Beziehung zu allgemein soziologischen Theorien. Daruber hinaus werden neue medizinsoziologische Entwicklungen in den Blick genommen.

Gunnar Stoltberg ist Professor filr Soziologie an der Universität Bielefeld. Er hat u.a. zur sozialen Konstruktion von Krankheit, der sog.

Alternativen Medizin sowie unter einer historischen Perspektive zu Patientemwelten und zur Binnendifferenzierung in Krankenhäusern publiziert

\section{Frubjahr 2001}

ca. 100 Seiten, kart, 19,80 DM ISBN $3-933127-26-2$

\section{LUDGER PRIES}

\begin{tabular}{l} 
Internationale \\
\hline Migration
\end{tabular}

Die vorubergehende oder dauerhafte Migration über die geographischen Grenzen sozialer und politischer Einheiten hinweg ist so alt wie die Menschheit selbst. In Deutschland bekamen grenzüberschreitende Wanderungs prozesse mit den tiefgreifenden Veränderungen in Osteuropa und mit der Stabilisierung der Europäischen Union eine neue Bedeutung. Gemessen an dieser veränderten Wirklichkeit hat die sozialwissenschaftliche Migrationsforschung in Europa und speziell in Deutschland noch sehr viel aufzuholen. Der Band gibt einen tberblick tuber wichtige klassische und neue Theorien internationaler Migration, wobei besonderes Gewicht auf aktuelle Forschungen aus Nordamerika gelegt wird:

\section{Ludger Pries íst Professor fur} Organisationssoziologie und Mitbestimmungsforschung an der Ruhr-Universität Bochum

Fruhiahr 2001

84 Seiten, kart., 18,80 DM

ISBN $3-933127 \cdot 27-0$
Bereits erschienen:

- Gesellschaftliche Differenzierung

- Neo-Institutionalismus

- Poststrukturalistische Soziologien

- Rísiko

- Religionssoziologie

- Weltgesellschaft

- Wissenssoziologie
Mühlenstraße 47 33607 Bielefeld

Fon 0521.63454

Fax 0521.61040

live@transcript-verlag.de

Besuchen Sie auch das Einsichten-Leserforum auf unserer Website! www.transcript-verlag.de

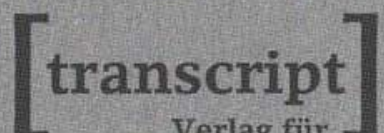

Verlag für $=$ Kommunikation,

Kultur und soziale Praxis 